\title{
Calibration Method to Eliminate Zeroth Order Effect in Lateral Shearing Interferometry
}

\author{
Chao FANG ${ }^{1,2^{*}}$, Yang XIANG ${ }^{1}$, Keqi $\mathrm{QI}^{1,2}$, and Dawei $\mathrm{CHEN}^{3}$ \\ ${ }^{1}$ State Key Laboratory of Applied Optics, Changchun Institute of Optics, Fine Mechanics and Physics, Chinese Academy \\ of Sciences, Changchun 130033, China \\ ${ }^{2}$ University of Chinese Academy of Sciences, Beijing 100039, China \\ ${ }^{3}$ Jilin University, Changchun 130012, China \\ *Corresponding author: Chao FANG_ E-mail: ciompfangchao@126.com
}

\begin{abstract}
In this paper, a calibration method is proposed which eliminates the zeroth order effect in lateral shearing interferometry. An analytical expression of the calibration error function is deduced, and the relationship between the phase-restoration error and calibration error is established. The analytical results show that the phase-restoration error introduced by the calibration error is proportional to the phase shifting error and zeroth order effect. The calibration method is verified using simulations and experiments. The simulation results show that the phase-restoration error is approximately proportional to the phase shift error and zeroth order effect, when the phase shifting error is less than $2^{\circ}$ and the zeroth order effect is less than 0.2 . The experimental result shows that compared with the conventional method with 9-frame interferograms, the calibration method with 5 -frame interferograms achieves nearly the same restoration accuracy.
\end{abstract}

Keywords: Calibration; phase measurement; phase shifting interferometry

Citation: Chao FANG, Yang XIANG, Keqi QI, and Dawei CHEN, "Calibration Method to Eliminate Zeroth Order Effect in Lateral Shearing Interferometry," Photonic Sensors, 2018, 8(3): 255-262.

\section{Introduction}

The lithography lens is an essential component of the lithography machine. The measurement of system wavefront aberration for the projection lens is one of the core technologies for the development of lithography [1-4]. There are several methods to measure system wavefront aberration, such as grating lateral shearing interferometry [5-8] and point diffraction interferometry [9-12]. Compared with the point diffraction interferometry, it is easy to realize the ultra-high precision measurement of high numerical aperture (NA) projection lens with grating lateral shearing interferometry. As a result, the grating lateral shearing interferometry is a promising technology for system wavefront aberration measurement [13].

In grating lateral shearing interferometry, the undesired zeroth order diffracted beam will reach the detector, causing the zeroth order effect error. Furthermore, the error is not negligible for wavefront aberration measurement of lithography lens. In 2004, Zhu et al. proposed a 9-frame phase shifting algorithm to solve the zeroth order effect

Received: 4 February 2018 / Revised: 14 March 2018

(C) The Author(s) 2018. This article is published with open access at Springerlink.com

DOI: $10.1007 / \mathrm{s} 13320-018-0500-2$

Article type: Regular 
problem under the condition of small phase shifting error [14]. In 2013, we improved the 11-frame algorithm to solve this problem under the condition of large phase shifting error [15]. The above mentioned solutions are achieved by designing a special phase shifting algorithm to suppress the zero order effect. These solutions increase the complexity of the phase shifting algorithm and require the collection of more frames of interferograms. It takes a longer time to collect more interferograms when more random errors, such as temperature, pressure, and vibration, are introduced. Moreover, it also increases the phase shifting amount, which requires design and fabrication of more complex phase shifter.

In this paper, we propose a calibration method to eliminate the zeroth order effect in lateral interferometry. A procedure of phase shifting calibration is performed before the procedure of phase shifting interferometry. The calibration procedure quantitatively calculates the zeroth order effect to eliminate its influence and thus reduce the complexity of the specially designed phase shifting algorithm. The principle of calibration is analyzed theoretically, and the analytical expression of the calibration error function is deduced. The relationship between the phase-restoration error and the calibration error is established. Finally, the simulations and experiment are described.

\section{Principles}

A typical grating lateral shearing interferometer is shown in Fig. 1. Grating divides the incident beam into diffracted beams. The \pm 1 st order diffracted beam passes through a spatial filter, and the 0th and higher order diffracted beams are eliminated. However, there is still a certain percentage of the 0th order beam reaching the charge-coupled device (CCD) detector that causes the zeroth order effect error.

Therefore, the error of the zeroth order effect must be eliminated before phase shifting interferometry. The method to eliminate the zeroth order effect is mainly achieved in two procedures. One procedure is the constant calculation during phase shifting calibration, and the other one is the removal of the zeroth order effect during phase shifting interferometry. The calibration constant is calculated once for one tested lens.

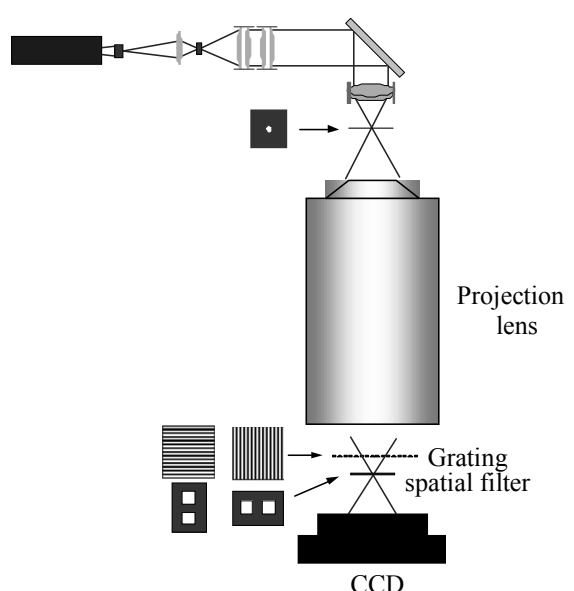

Fig. 1 Block diagrams of grating lateral shearing interferometer

\subsection{Calculation of calibration constant}

We propose a 9-step phase shifting calibration method to calculate the calibration constant to quantify the zeroth order effect. The phase shifting amount for each step is $\pi / 2$, then the phase shifting amount in the calibration procedure can be expressed as

$$
\phi_{n}=n \frac{\pi}{2}
$$

where $n(n=-4,-3, \cdots, 0, \cdots, 3,4)$ is the number of step.

The 0th and \pm 1 st order beams interfere with each other in the calibration procedure. The fringe intensity distribution of $I_{n}$ for the $n$-th step is

$$
\begin{aligned}
I_{n}= & Q+V_{1} \cos \left(\theta_{1}+\phi_{n}\right)+ \\
& V_{2} \cos \left(\theta_{2}+\phi_{n} / 2\right)+V_{3} \cos \left(\theta_{3}+\phi_{n} / 2\right)
\end{aligned}
$$

where $\theta_{1}$ is an unknown phase needing to be restored, $\theta_{2}$ and $\theta_{3}$ are undesired phases distorted by the 0 th order beam, $V_{1}$ is the intensity modulation of the tested interference, and $V_{2}$ and $V_{3}$ are the intensity 
modulations of the undesired interference.

In order to calculate the zeroth order effect, the calibration constants of $K_{1}$ and $K_{2}$ are defined as follows:

$$
\left\{\begin{array}{c}
K_{1}=V_{2} \sin \theta_{2}+V_{3} \sin \theta_{3} \\
K_{2}=V_{2} \cos \theta_{2}+V_{3} \cos \theta_{3} .
\end{array}\right.
$$

According to (1) to (3), the calibration constants can be calculated as follows:

$$
\left\{\begin{array}{c}
K_{1}=-\frac{\sqrt{2}}{4}\left(I_{1}-I_{-1}\right)-\frac{\sqrt{2}}{4}\left(I_{3}-I_{-3}\right) \\
K_{2}=-\frac{1}{4}\left(I_{4}+I_{-4}\right)-2 I_{0} .
\end{array}\right.
$$

\subsection{Removal of the zeroth order effect}

In the phase shifting interferometry procedure, the intensity distribution of $I_{j}$ for the $j$-th step is

$$
\begin{aligned}
I_{j}= & Q+V_{1} \cos \left(\theta_{1}+\varphi_{j}\right)+ \\
& V_{2} \cos \left(\theta_{2}+\varphi_{j} / 2\right)+V_{3} \cos \left(\theta_{3}+\varphi_{j} / 2\right)
\end{aligned}
$$

where $\varphi_{j}$ is phase shifting amount in the interferometry.

The intensity distribution of the zeroth order effect to be calibrated is represented as follows:

$$
\begin{aligned}
I_{c j} & =V_{2} \cos \left(\theta_{2}+\varphi_{j} / 2\right)+V_{3} \cos \left(\theta_{3}+\varphi_{j} / 2\right) \\
& =K_{2} \cos \left(\varphi_{j} / 2\right)-K_{1} \sin \left(\varphi_{j} / 2\right) .
\end{aligned}
$$

After calibrating $K_{1}$ and $K_{2}$, the zeroth order effect with corresponding phase shifting amount can be calculated according to (6). The intensity distribution of each frame after removing the error of the zeroth order effect can be expressed as follows:

$$
I_{t j}=I_{j}-I_{c j}=Q+V_{1} \cos \left(\theta_{1}+\varphi_{j}\right)
$$

From (7), we conclude that the intensity distribution is not affected by the zeroth order effect. The calibration and removal of the zeroth order effect are achieved.

\section{Error analyses}

\subsection{Intensity distribution calibration error}

Owing to the manufacturing error and alignment error of micro displacement stage and grating, the phase shifting error always exists in the phase shifting calibration procedure. The phase shifting amount for $n$-frame is

$$
\phi_{n}=n \cdot \frac{\pi}{2}+n \cdot \mathcal{\varepsilon}
$$

where $\varepsilon$ is the phase shifting error.

We substitute (8) into (4) and express (4) as two sets of Taylor expansions for the phase shifting error of $\varepsilon$, ignoring the higher order of $\varepsilon^{2}$ or more. The calibration constants are approximated as follows:

$$
\begin{aligned}
K_{1}^{\prime} & \approx V_{2}\left(1-\frac{1}{2} \varepsilon\right) \sin \theta_{2}+V_{3}\left(1-\frac{1}{2} \varepsilon\right) \sin \theta_{3} \\
& =\left(1-\frac{1}{2} \varepsilon\right) K_{1} \\
& K_{2}^{\prime} \approx V_{2} \cos \theta_{2}+V_{3} \cos \theta_{3}=K_{2} .
\end{aligned}
$$

In the phase shifting interferometry, the intensity distribution of the zeroth order effect with phase shifting calibration error can be expressed as follows:

$$
\begin{aligned}
I_{c j}^{\prime} & =K_{2}^{\prime} \cos \left(\varphi_{j} / 2\right)-K_{1}^{\prime} \sin \left(\varphi_{j} / 2\right) \\
& =I_{c j}+\frac{\varepsilon}{2} K_{1} \sin \left(\varphi_{j} / 2\right) .
\end{aligned}
$$

The intensity distribution calibration error of the zeroth order effect is expressed as follows:

$$
\Delta I_{c j}=I_{c j}^{\prime}-I_{c j}=\frac{\varepsilon}{2} K_{1} \sin \left(\varphi_{j} / 2\right)
$$

In general, the intensities of the \pm 1 order beams are equal, therefore, $V_{2}=V_{3}$. The intensity distribution calibration error is expressed as

$$
\Delta I_{c j}=\varepsilon V_{2} A
$$

where $A=\left(\sin \theta_{2}+\sin \theta_{3}\right) \sin \left(\varphi_{j} / 2\right) / 2 \quad$ is $\quad$ a constant.

From (13), we conclude that the intensity distribution calibration error of the zeroth order effect is proportional to the phase shifting error and the zeroth order effect. As the phase shifting error increases, the calibration error increases linearly. As the zeroth order effect increases, the calibration error increases linearly.

\subsection{Phase-restoration error}

The intensity distribution calibration error is 
shown as the gray of the interferogram, which is an intermediate quantity in the measurement. The final result of the measurement is phase. Therefore, it is necessary to establish the relationship between the phase-restoration error and the calibration error.

The intensity distribution of $I_{t j}^{\prime}$ affected by calibration error is denoted as follows:

$$
I^{\prime}{ }_{t j}=I_{t j}-\Delta I_{c j} .
$$

Here, the widely used Hariharan algorithm is used to illustrate the method to establish the relationship between the phase-restoration error and calibration error [16]. The phase shifting amount for each step of the Hariharan algorithm is $\pi / 2$. The Hariharan algorithm is expressed as

$$
\tan \theta=\frac{2\left(I_{1}-I_{-1}\right)}{\left(I_{2}+I_{-2}\right)-2 I_{0}}
$$

where $\theta$ is the phase to be restored.

The phase with the calibration error is expressed as

$$
\tan \theta^{\prime}=\frac{2\left(I_{1 t}-I_{-1 t}\right)-\sqrt{2} \varepsilon K_{1}}{\left(I_{2 t}+I_{-2 t}\right)-2 I_{0 t}}=\tan \theta\left(1-2 \varepsilon V_{2} B\right)
$$

where

$$
B=\frac{\sqrt{2}}{2} \frac{\left(\sin \theta_{2}+\sin \theta_{3}\right)}{\left(I_{t 2}+I_{t-2}\right)-2 I_{t 0}}
$$

and $B$ is a constant.

Considering that the phase-restoration error of $\Delta \theta$ is small, the phase-restoration error is as follows:

$$
\begin{aligned}
\Delta \theta & =\theta-\theta^{\prime} \approx \tan \left(\theta-\theta^{\prime}\right) \\
& =\varepsilon V_{2} B \sin 2 \theta .
\end{aligned}
$$

From (18), the phase-restoration error introduced by the calibration error of the zeroth order effect varies as the sine of double phase, and the worst condition is at $\pi / 4$. The phase-restoration error is also proportional to the phase shifting error and zeroth order effect. As the phase shifting error increases, the phase-restoration error increases linearly. As the zeroth order effect increases, the phase-restoration error increases linearly.

\section{Simulations}

In this section, a lateral shearing interferometry affected by the zeroth order effect is simulated to verify the analyses. The wavefront with common aberrations locates in unit circle. The wavefront is sheared in the $x$ direction, the shearing ratio is 0.1 , and the zeroth order effect is $V_{2}=V_{3}=12.5 \% V_{1}$. The shearing phase is shown in Fig. 2.

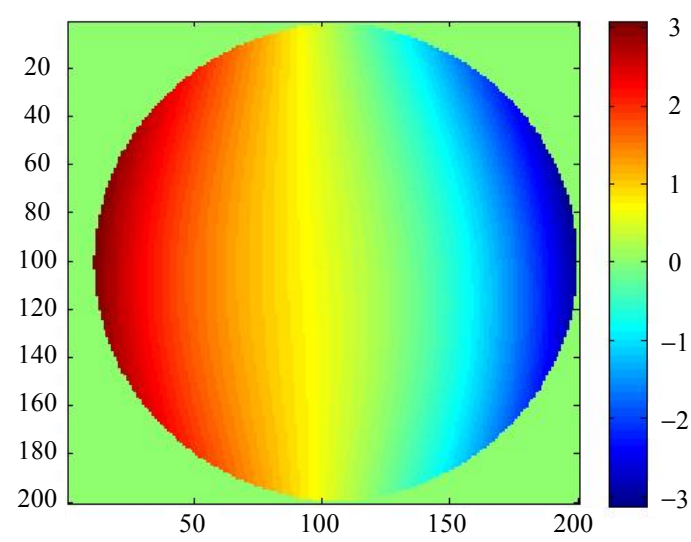

Fig. 2 Shearing phase in the simulation.

According to the calibration method, under the conditions of $\varepsilon=1^{\circ}$ and $V_{2}=V_{3}=12.5 \% V_{1}$, the phase shifting amount of each step is $\pi / 2$, and a sequence of 9-frame interferogram is obtained in the calibration procedure. According to (4), the calibration constants $K_{1}$ and $K_{2}$ are calculated. When $j=-2$, the calibration result of the zeroth order effect is shown in Fig. 3.

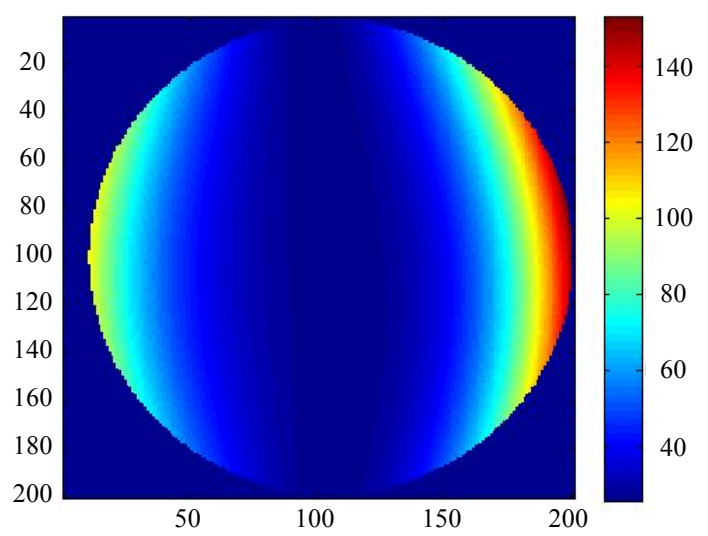

Fig. 3 Calibration results of the zeroth order effect when $j=-2$.

Depending on the calibration constants, the phase is restored using the Hariharan algorithm in 
the interferometry procedure. The restoration phase is illustrated in Fig. 4.

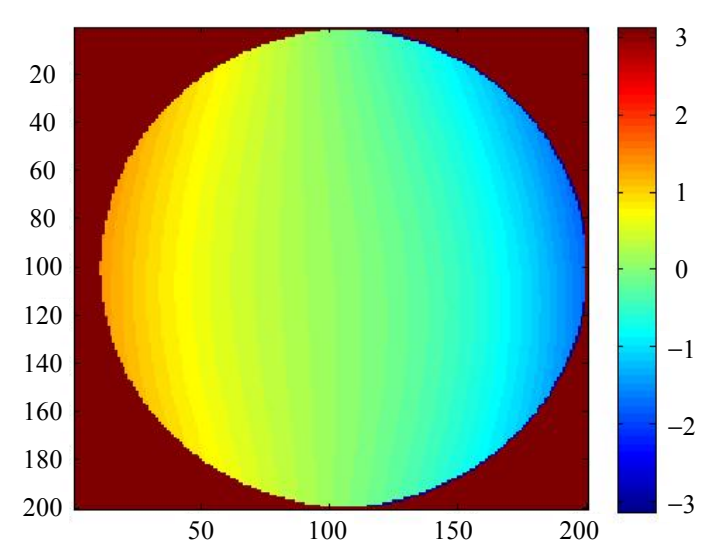

Fig. 4 Restoration phase after removing the zeroth order effect.

By comparing the results of Figs. 2 and 4, the phase-restoration error is obtained, as shown in Fig. 5. The root mean square (RMS) of phase-restoration error is $0.001598 \mathrm{rad}$. Under the same condition, the RMS of phase-restoration error is 0.001509 rad with the 9-frame phase shifting algorithm proposed by Zhu [14]. Two different methods are used to deal with the interferograms with the zeroth order effect, and the relative error of the phase-restoration error is less than $6 \%$. Therefore, the calibration method is expected to be available.

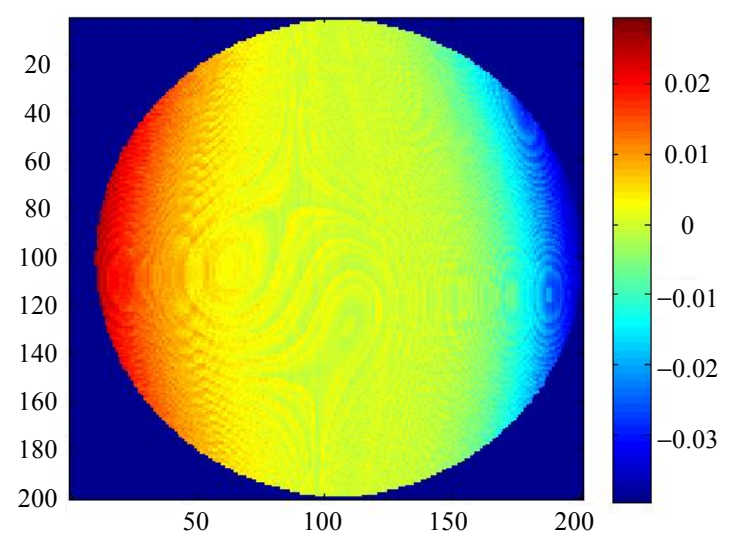

Fig. 5 Phase-restoration error caused by the calibration error of the zeroth order effect.

Under the condition of $V_{2}=V_{3}=12.5 \% V_{1}$, simulations with different phase shifting errors are carried out, respectively. We linearly fit the relationship between the phase-restoration error and the phase shifting error, as shown in Fig. 6. The fit result shows the coefficient of determination is 0.9794 , which is closed to 1 . Therefore, when the phase shifting error is less than $2^{\circ}$, the phase-restoration error approximately increases with the phase shifting error linearly, that is consistent with the theoretical analysis in Section 3.

Under the condition of $\varepsilon=1^{\circ}$, simulations with different zeroth order effects are carried out. We linearly fit the relationship between the phase-restoration error and the zeroth order effect, as shown in Fig. 7. The fit result shows the coefficient of determination is 0.9888 , which is close to 1 . Therefore, when the zeroth order effect is less than 0.2 , the phase-restoration error increases with the zeroth order effect approximately linearly, that is consistent with the theoretical analysis in Section 3.

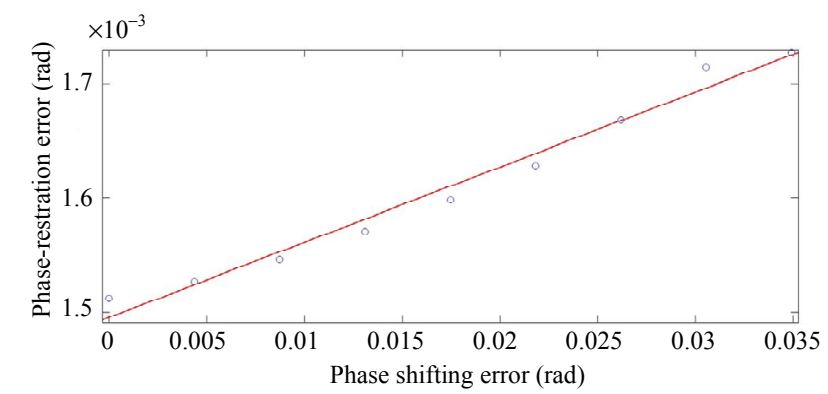

Fig. 6 Relationship between the phase-restoration error and phase shifting error.

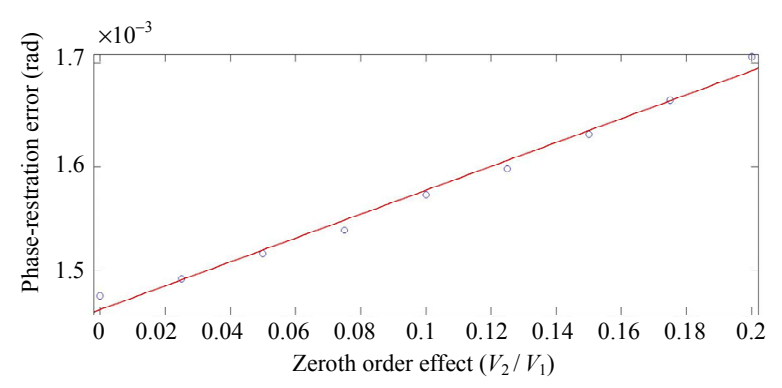

Fig. 7 Relationship between the phase-restoration error and the zeroth order effect.

\section{Experiments}

The experimental grating lateral shearing interferometer is shown in Fig. 8. 


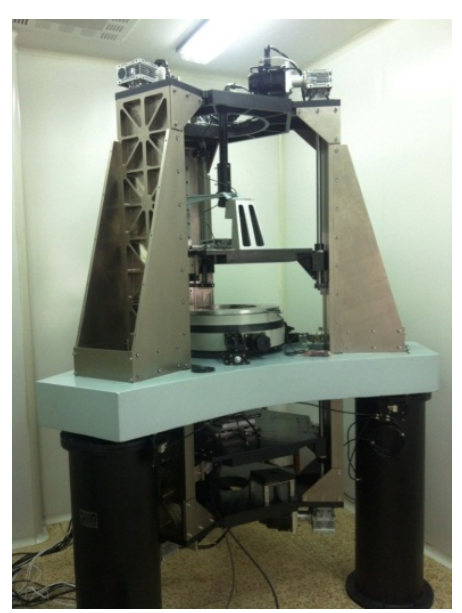

Fig. 8 Experimental lateral shearing interferometer.

With the calibration method described in Section 3 , the restoration phase is shown in Fig. 9 by using the experimental interferometer. With the conventional 9-frame phase shifting algorithm, the restoration phase is shown in Fig. 10. The difference between the two restoration phases is shown in Fig. 11.

With the calibration method, the RMS of the phase with the calibration method is $5.5342 \mathrm{rad}$. With the 9-frame phase shifting algorithm, the RMS of the phase is $5.5346 \mathrm{rad}$. We compare the results of the two phases point by point, the RMS of the phase difference is 0.1134 rad. Relative error of the RMS is less than $2.5 \%$. We perform 10 experiments under the same experimental conditions. The experimental results are shown in Fig. 12. The average relative error is $2.07 \%$, and the RMS of relative error is $0.01 \%$.

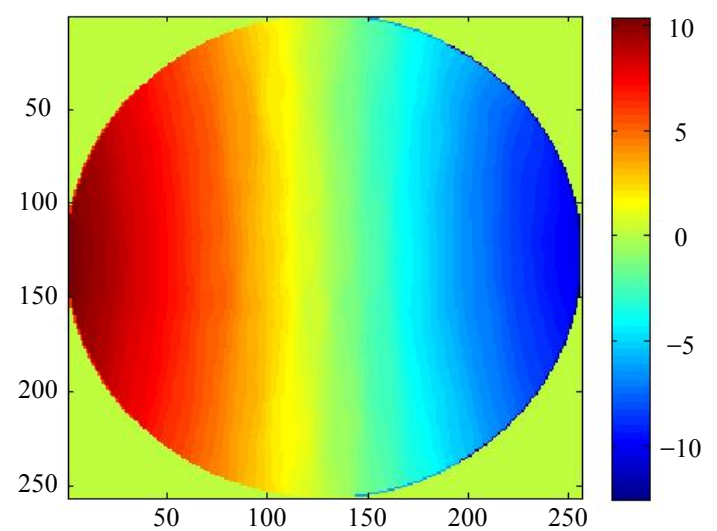

Fig. 9 RMS of the restoration phase with the calibration method.

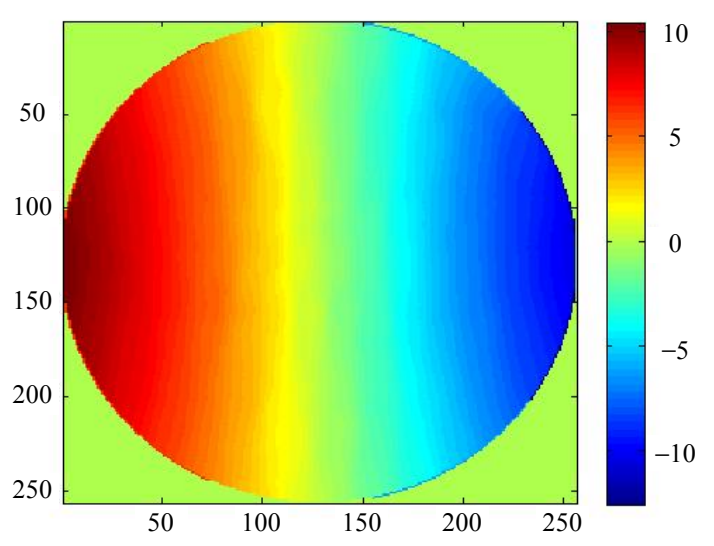

Fig. 10 RMS of the restoration phase with the conventional 9-frame phase shifting algorithm.

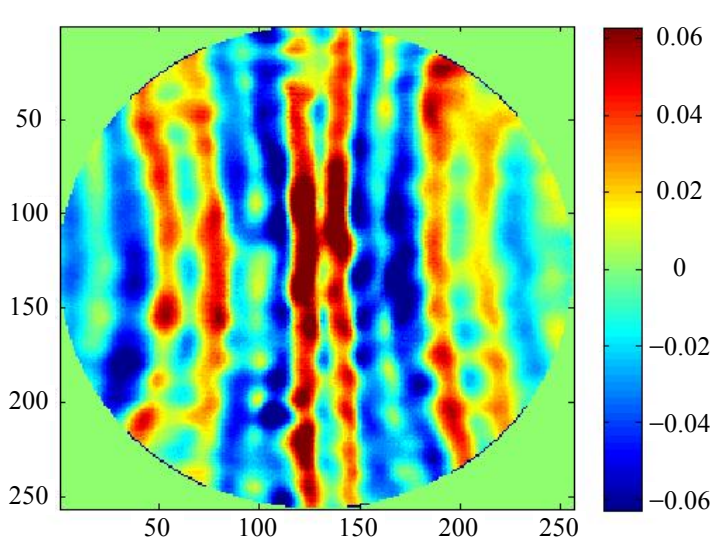

Fig. 11 RMS of the restoration phase difference with two methods.

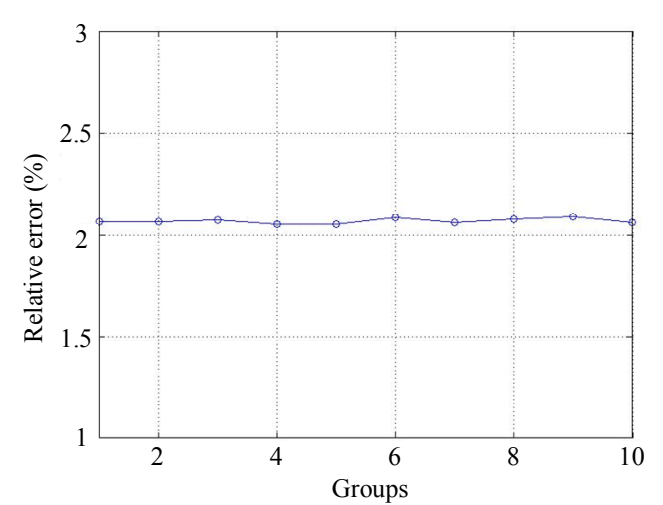

Fig. 12 Relative errors with 10 experiments.

Compared the conventional method with 9-frame interferograms, the calibration method with 5-frame interferograms achieve nearly the same restoration accuracy. Hence, the calibration method is available to eliminate the zeroth 
order effect.

\section{Conclusions}

A calibration method to eliminate the zeroth order effect is proposed in the paper. The principle of calibration is analyzed theoretically, and the analytical expression of the calibration error caused by the phase shifting error is deduced. Using the Hariharan algorithm, the relationship between the phase-restoration error and calibration error is established. The results show that the calibration error of intensity distribution is proportional to the phase shifting error and zeroth order effect. The phase-restoration error introduced by the calibration error of the zeroth order effect is also proportional to the phase shifting error and zeroth order effect. The calibration method is verified by carrying out simulations and experiments. The simulation results show that the phase-restoration error is approximately proportional to the phase shift error and zeroth order effect, when the phase shifting error is less than $2^{\circ}$ and the zeroth order effect is less than 0.2. Simulations are consistent with the theoretical analysis. The experimental result shows that compared with the conventional method with 9-frame interferograms, the calibration method with 5-frame interferograms achieves nearly the same restoration accuracy. Therefore, the calibration method is available to eliminate the zeroth order effect.

\section{Acknowledgment}

This work was supported by the National S\&T (Science and Technology) Special Program (No. 2009ZX02202005).

Open Access This article is distributed under the terms of the Creative Commons Attribution 4.0 International License (http://creativecommons.org/licenses/by/4.0/), which permits unrestricted use, distribution, and reproduction in any medium, provided you give appropriate credit to the original author(s) and the source, provide a link to the Creative Commons license, and indicate if changes were made.

\section{References}

[1] Y. Q. Yu, Y. F. Zhang, Z. L. Ou, X. Chen, Q. D. Huang, and S. C. Ruan, "Simultaneous measurement of one dimensional bending and temperature based on Mach-Zehnder interferometer," Photonic Sensors, 2015, 5(4): 376-384.

[2] L. C. Zhang, X. K. Cai, and G. Shi, "Optical coatings for DUV lithography," Chinese Optics, 2015, 8(2): 169-181.

[3] S. T. Gao, D. C. Wu, and E. L. Miao, "Distortion correcting method when testing large-departure asphere," Chinese Optics, 2017, 10(3): 383-390.

[4] F. Wang, X. Z. Wang, M. Y. Ma, D. Q. Zhang, W. J. Shi, and J. M. Hu, "Aberration measurement of projection optics in lithographic tools by use of an alternating phase-shifting mask," Applied Optics, 2006, 45(2): 281-287.

[5] F. Z. Dai, J. Li, X. Z. Wang, and Y. Bu, "Exact two-dimensional zonal wavefront reconstruction with high spatial resolution in lateral shearing interferometry," Optics Communications, 2016, 367: 264-273.

[6] M. Takeda and S. Kobayashi, "Lateral aberration measurements with a digital Talbot interferometer," Applied Optics, 1984, 23(11): 1760-1764.

[7] M. Hasegawa, C. Ouchi, T. Hasegawa, S. Kato, A. Ohkubo, A. Suzuki, et al., "Recent progress of EUV wave-front metrology in EUVA," SPIE, 2004, 5533: 27-36.

[8] H. Schreiber and J. Schwider, "Lateral shearing interferometer based on two Ronchi gratings in series," Applied Optics, 1997, 36(22): 53215324.

[9] F. Z. Bai, X. Q. Wang, K. Z. Huang, and F. Tian, "Analysis of spatial resolution and pinhole size for single-shot point-diffraction interferometer using in closed-loop adaptive optics," Optics Communications, 2013, 297(12): 27-31.

[10] S. H. Lee, P. Naulleau, K. A. Goldberg, F. Piao, W. Oldham, and J. Bokar, "Phase-shifting point-diffraction interferometry at $193 \mathrm{~nm}$," Applied Optics, 2013, 39(31): 5768-5772.

[11] K. A. Goldberg, P. Naulleau, P. Denham, and E. H. Anderson, "EUV interferometric testing and alignment of the 0.3-NA MET optic," SPIE, 2004, 5374(7): 64-73.

[12] P. Gao, I. Harder, V. Nercissian, K. Mantel, and B. Yao, "Phase-shifting point-diffraction interferometry with common-path and in-line configuration for microscopy," Optics Letters, 2010, 35(5): $712-714$. 
[13] R. Miyakawa, P. Naulleau, and K. Goldberg, "Analysis of systematic errors in lateral shearing interferometry for EUV optical testing," SPIE, 2009, 7272: 1-6.

[14] Y. C. Zhu, K. Sugisaki, C. Ouchi, M. Hasegawa, M. Niibe, A. Suzuki, et al., "Lateral shearing interferometer for EUVL: theoretical analysis and experiment," in Proceeding of Conference on Emerging Lithographic Technologies VIII,
Santa Clara, CA, USA, 2004, pp. 824832.

[15] C. Fang, Y. Xiang, K. Q. Qi, C. L. Zhang, and C. S. $\mathrm{Yu}$, "An 11-frame phase shifting algorithm in lateral shearing interferometry," Optics Express, 2013, 21(23): 28325-28333.

[16] P. Hariharan, "Digital phase-stepping interferometry: effects of multiply reflected beams," Applied Optics, 1987, 26(13): 2506-2507. 